\title{
Epilepsy management in pregnant HIV+ women in sub-Saharan Africa, clinical aspects to consider: a scoping review
}

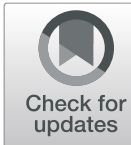

Sonia Menon ${ }^{1 *}$, Lenka Benova ${ }^{1}$ and Hillary Mabeya ${ }^{2}$

\begin{abstract}
Background: Since the introduction of highly active antiretroviral therapy (HAART), acquired immune deficiency syndrome (AIDS) related mortality has markedly declined. As HAART is becoming increasingly available, the infection with human immunodeficiency virus (HIV+) in sub-Saharan Africa (SSA) is becoming a chronic condition. While pregnancy in HIV+ women in SSA has always been considered a challenging event for the mother and the fetus, for pregnant HIV+ women also diagnosed with epilepsy (WWE), there are additional risks as HIV increases the odds of developing seizures due to the vulnerability of the central nervous system to other infections, immune dysfunction, and overall metabolic disturbances. In light of a growing proportion of HIV+ WWE on HAART and an increasing number of pregnant women accessing mother-to-child transmission of HIV programs through provision of HAART in SSA, there is a need to develop contextualized and evidenced-based clinical strategies for the management of epilepsy in this population. In this study, we conduct a literature scoping review to identify issues that warrant consideration for clinical management.

Result: Twenty-three articles were retained after screening, which covered six overarching clinical aspects: status epilepticus (SE), Stevens-Johnson syndrome/toxic epidermal necrolysis (SJS/TEN), dyslipidemia, congenital malformation (CM), chronic kidney disease (CKD), and neurological development. No studies for our population of interest were identified, highlighting the need for a cautionary approach to be employed when extrapolating findings.

Conclusion: High risks of CM and drug interactions with first-line antiepileptic drugs (AEDs) warrant measures to increase the accessibility and choices of safer second-line AEDs. To ensure evidence-based management of epilepsy within this population, the potential high prevalence of SE, CKD, dyslipidemia, and SJS/TEN and the cumulative effect of drug-drug interactions should be considered. Further understanding of the intersections between pregnancy and drug-drug interactions in SSA is needed to ensure evidenced-based management of epilepsy in pregnant HIV+ WWE. To prevent SE, the barriers for AED treatment adherence in pregnant HIV+ women should be explored. Our review underscores the need to conduct cohort studies of HIV+ WWE in reproductive age over time and across pregnancies to capture the cumulative effect of HAART and AED to inform clinical management.
\end{abstract}

Keywords: HIV, Epilepsy, Antiretroviral treatment, Antiepileptics, Pregnancy, Women of reproductive age

* Correspondence: soniasimonemenon@gmail.com

${ }^{1}$ Instiute of Tropical Medicine Antwerp, Antwerp, Belgium

Full list of author information is available at the end of the article

C C The Author(s). 2020 Open Access This article is licensed under a Creative Commons Attribution 4.0 International License, which permits use, sharing, adaptation, distribution and reproduction in any medium or format, as long as you give appropriate credit to the original author(s) and the source, provide a link to the Creative Commons licence, and indicate if changes were made. The images or other third party material in this article are included in the article's Creative Commons licence, unless indicated otherwise in a credit line to the material. If material is not included in the article's Creative Commons licence and your intended use is not permitted by statutory regulation or exceeds the permitted use, you will need to obtain permission directly from the copyright holder. To view a copy of this licence, visit http://creativecommons.org/licenses/by/4.0/. The Creative Commons Public Domain Dedication waiver (http://creativecommons.org/publicdomain/zero/1.0/) applies to the data made available in this article, unless otherwise stated in a credit line to the data. 


\section{Background}

According to the World Health Organization (WHO), there were approximately 38 million people across the globe with human immunodeficiency virus (HIV) or acquired immune deficiency syndrome (AIDS) at the end of 2019 [1]. The burden disproportionately affects subSaharan Africa (SSA) where over two thirds of all people living with HIV reside [1] and where women and girls account for $59 \%$ of all new HIV infections [2]. Since the introduction of highly active antiretroviral therapy (HAART), HIV is becoming a chronic condition for the 16.4 million people on the drug regimen in SSA [3]. Furthermore, around 1.4 million HIV infections among children were prevented between 2010 and 2018 due to the implementation of services for the prevention of mother-to-child transmission (PMTCT) of HIV [4].

Use of HAART has entailed an excess risk of seemingly unrelated non-AIDS conditions, such as hyperlipidemia [5], Stevens-Johnson syndrome/toxic epidermal necrolysis (SJS/TEN) [6], chronic kidney disease (CKD) [7], and cardiovascular diseases [8, 9]. Furthermore, $\mathrm{HIV}+$ individuals are at heightened risk for developing seizures due to the vulnerability of the central nervous system to opportunistic diseases, immune dysfunction, and metabolic disturbances [10]. In addition, some drugs indicated for the treatment of HIV or HIV-associated infections and coexisting metabolic derangements may also induce seizures [10]. A recent meta-analysis reported a high pooled prevalence of seizures in a general HIV+ population of 62 per 1000 (95\% confidential interval [CI] 37.3-93.1) [11], in contrast to a pooled lifetime prevalence of 7.60 per 1000 persons (95\% CI 6.17-9.38) reported in a general population [12].

Epilepsy is diagnosed after a person experiences two unprovoked seizures more than $24 \mathrm{~h}$ apart [13]. The majority of seizures in $\mathrm{HIV}+$ individuals are generalized seizures [14] involving both cerebral hemispheres, which may need emergency medical attention. With an increasing burden of chronic HIV infections in low-income countries (LIC), HIV infection has become an important risk factor of epilepsy and status epilepticus (SE) [15].

Nearly $80 \%$ of people with epilepsy live in LIC, where it is estimated that in an ideal scenario up to $70 \%$ of people living with epilepsy are seizure-free if properly diagnosed and treated [16].

In 2019, in HIC, there were estimated to be 49 per 100, 000 people diagnosed with epilepsy each year, whereas in low- to middle-income countries, estimates were at 139 per 100, 000 [17]. It is noteworthy that the epilepsy treatment gap, defined as the proportion of people with epilepsy who require treatment but do not receive it [18], in the general population varies from $23 \%$ in Senegal [19] to $100 \%$ in Uganda, Tanzania, Gambia, and Togo [18].
In most LIC, the first-line enzyme-inducing antiepileptic drug (EI-AED), phenobarbital, is the only AED in widespread use [20]. Although other first-line AEDs, including phenytoin, carbamazepine, and valproic acid, are commonly available, they entail higher out-of-pocket costs [21]. The first-line EI-AEDs, metabolized predominantly by the liver, are problematic for HIV+ individuals as they are inducers of the cytochrome P450 hepatic enzyme system and thus interact with anti-infective drugs, including HAART [10].

In 2018, the WHO issued interim guidance for HAAR $\mathrm{T}$ recommending two nucleoside reverse transcriptase inhibitors (NRTIs) tenofovir, lamivudine, paired with dolutegravir to replace nevirapine (NRTI) as the preferred first-line regimen for HIV treatment, also in adolescent girls and women who are pregnant [22]. However, dolutegravir effectiveness has been shown to be reduced in patients treated with phenobarbital [23], and there have also been reports of interactions with valproic acid [24]. This gave rise to the recommendation that to prevent virologic failure, concomitant use of HAART regimens that include protease inhibitors (PI) or NRTIs and EI-AED should be avoided [25].

Compared to these EI-AEDs, second-line broadspectrum non-EI-AEDs including levetiracetam and lamotrigine have fewer drug interactions [26]. However, these second-line AEDs for generalized and partial seizures are not widely available in SSA [20]. Currently, the only second-line AED for partial and generalized seizures for pregnant women in the WHO Essential List of Medicines is lamotrigine [27], which undergoes extensive metabolism in the liver [28].

Apart from drug interactions with HAART and antiinfective agents, first-line EI-AEDs are significantly more teratogenic than second-line AEDs [29]. The WHO recommends carbamazepine for pregnant women with epilepsy (WWE) if no second-line AED is available [17], due to its slightly lower prevalence of congenital malformation (CM) (5.5\%), compared to phenobarbital $(6.5 \%)$, and lamotrigine and levetiracetam $(2.9 \%$ and $2.8 \%$, respectively) [30] (Table 1).

When treating WWE, clinicians face the additional dilemma that valproic acid, a first-line AED recommended for HIV+ individuals in LIC as it is not enzyme inducing [10], is associated with the highest prevalence of CM $(10.3 \%)$ [30]. In addition, given the geographic overlap with other endemic diseases, a non-negligible proportion of pregnant $\mathrm{HIV}+$ WWE may concomitantly receive treatment with other potentially teratogenic drugs, including anti-TB drugs [32]. Therefore, epilepsy management during pregnancy, particularly in $\mathrm{HIV}+$ women, requires an equilibrium between seizure control, viral load control, co-infection prevention, and risk minimization for the WWE and their fetuses [33]. 
Table 1 Table with safety profile of AED in pregnant women

\begin{tabular}{|c|c|c|c|c|c|c|}
\hline AED & Indication & $\begin{array}{l}\text { Neurological development after in } \\
\text { utero exposure }\end{array}$ & $\begin{array}{l}\% \text { of fetal } \\
\text { malformation }\end{array}$ & Enzyme inducing & SJS/TEN & Dyslipidemia \\
\hline Phenobarbital & $\begin{array}{l}\text { Broad spectrum } \\
\text { AED }\end{array}$ & $\begin{array}{l}\text { No statistically significant association } \\
\text { with adverse neurological outcomes } \\
\text { and autism }\end{array}$ & $6.50 \%^{2}$ & Yes & Yes & Yes \\
\hline Phenytoin & $\begin{array}{l}\text { Broad spectrum } \\
\text { AED }\end{array}$ & $\begin{array}{l}\text { No statistically significant association } \\
\text { with adverse neurological outcomes } \\
\text { and autism }{ }^{1}\end{array}$ & $6.4 \%^{2}$ & Yes & Yes & Yes \\
\hline Carbamazepine & $\begin{array}{l}\text { Broad spectrum } \\
\text { AED }\end{array}$ & $\begin{array}{l}\text { No statistically significant association } \\
\text { with adverse neurological outcomes } \\
\text { and autism }{ }^{1}\end{array}$ & $5.50 \%^{2}$ & Yes & Yes & Yes \\
\hline Valproic acid & $\begin{array}{l}\text { Broad spectrum } \\
\text { AED }\end{array}$ & $\begin{array}{l}\text { Pooled OR of adverse } \\
\text { neurodevelopmental outcomes and } \\
\text { autism compared to women who } \\
\text { did not receive AED during } \\
\text { pregnancy (OR=7.40; } \mathrm{Cl}: 95 \% 3.00 \text { to } \\
\text { 18.46); (OR:17.29; } 95 \% \mathrm{Cl} \text { : 2.40-217.60) } \\
\text { respectively }{ }^{1}\end{array}$ & $10.30 \%^{2}$ & No & Yes & No evidence \\
\hline Second line AED & & & $\begin{array}{l}\text { Absence of AED } \\
\text { exposure: } 2.6 \%{ }^{2}\end{array}$ & & & \\
\hline Lamotrigine & $\begin{array}{l}\text { Broad spectrum } \\
\text { AED }\end{array}$ & $\begin{array}{l}\text { Pooled OR of autism compared to } \\
\text { women who did not receive AED } \\
\text { during pregnancy (OR: } 8.88 ; 95 \% \mathrm{Cl} \text { : } \\
1.28-112.00)^{1}\end{array}$ & $2.90 \%^{2}$ & No & Yes & No evidence \\
\hline Levetiracetam & $\begin{array}{l}\text { Broad spectrum } \\
\text { AED }\end{array}$ & $\begin{array}{l}\text { No statistically significant association } \\
\text { with adverse neurological outcomes } \\
\text { and autism }\end{array}$ & $2.80 \%^{2}$ & $\begin{array}{l}\text { No } \\
\text { Not enzyme inducing, } \\
\text { not associated with clinically } \\
\text { relevant drug interactions }\end{array}$ & $\begin{array}{l}\text { No } \\
\text { evidence }\end{array}$ & No evidence \\
\hline
\end{tabular}

${ }^{1}$ Veroniki, et al. [31]. Published 2017 Jul 20. doi:10.1136/bmjopen-2017-017248

${ }^{2}$ Tomson et al. [30]

With a large number of HIV+ women who will be on HAART prior to getting pregnant and HIV+ pregnant women embarking on PMTCT programs, there is a need to develop evidenced-based clinical guidelines on epilepsy management in pregnant HIV+ women. This review seeks to identify aspects to be considered in clinical management of epilepsy in pregnant HIV+ women in SSA. Specifically, it explores clinical aspects which intersect between HIV, HAART, AED, and pregnancy.

\section{Methods}

The methods were organized according to the seven following stages using a methodological framework for scoping reviews [34].

\section{Search strategy}

A scoping review was performed to characterize the current understanding about the management of epilepsy in pregnant HIV+ women SSA. Literature searches were performed on PubMed on August 4, 2020. January 1997 onwards was selected as a starting date to limit the publications to the combined HAART era. Different sets of keywords and Boolean terms were used to identify literature, e.g., dyslipidemia AND pregnan* AND antiepileptic drugs AND antiretroviral AND Africa; dyslipidemia AND antiretroviral therapy AND pregnan* AND Africa; dyslipidemia
AND antiretroviral therapy AND Africa; dyslipidemia AND HIV AND Africa (Additional file 1: Annex 1). Reference lists from retrieved publications were reviewed to identify additional manuscripts not captured by the search.

Literature reporting on the pooled burden pertaining to all six clinical aspects explored in this review was searched for our population of interest, along with risk factors. If no studies were identified for this specific population, a stepwise search process was undertaken, omitting one key term in each subsequent search. An additional hand-search was performed on the reference list of the retrieved literature.

\section{Inclusion criteria}

Inclusion criteria are as follows: observational prospective, retrospective, and cross-sectional studies; quasi- and randomized controlled trials (RCTs); pre- and poststudies without a control group; systematic reviews; and/ or meta-analyses reflecting the most current pooled evidence.

\section{Exclusion criteria}

Exclusion criteria are as follows: case studies; case series; commentaries; studies included within the most current pooled evidence; studies focusing on children, older adults, and HAART-naïve patients; studies whose 
primary endpoints are not the ones of interest; and nonEnglish literature.

\section{Research question}

This scoping review aims to answer the following question: What are the clinical aspects which should be considered to provide safe and effective clinical management of pregnant HIV+ WWE on HAART in SSA?

\section{Study selection}

We used the PICO model to answer our research question.

\section{Population}

Pregnant HIV+ WWE in SSA.

\section{Intervention}

The use of HAART and AEDs in pregnant HIV+ WWE.

\section{Comparison}

$\mathrm{HIV}+\mathrm{WWE}$ on AED, with or without a control group.

\section{Outcome(s)}

The outcomes are safety of the concomitant use of AED/HAART use in pregnant women, and adequate management of AED in pregnant $\mathrm{HIV}+\mathrm{WWE}$ on HAART.

\section{Extraction of data}

Microsoft Excel 2016 was used to gather information on author(s), year of publication, study location and population, study design, main exposure of interest and outcome, and key findings.

\section{Synthesis of findings}

Key findings were broken down into specific categories, derived from the articles rather than a predefined framework. We adhered to the Preferred Reporting Items for Systematic Reviews and Meta-analyses-Extension for Scoping Reviews (PRISMA-ScR) checklist and guidelines (Additional file 2: Annex 2).

\section{Results}

Using the broadest search terms, of the 581 articles retrieved, 23 studies were retained; these included 19 original research articles and 4 systematic reviews and/or meta-analyses (Fig. 1). No articles on pregnant HIV+ WWE on HAART and AEDs in SSA were retrieved; only one study on HIV+ individuals on HAART and AEDs in SSA was identified. We identified six overarching clinical aspects: SE, STJ/TEN, dyslipidemia, CM, CKD, and neurological development, which intersect with pregnancy, AED treatment, and HAART (Table 2).

\section{Status epilepticus}

There is no study examining AED adherence, in pregnant HIV + women on both HAART and AED.

SE, HIV, and pregnancy intersect. SE in LIC has an infectious etiology or in those with already established epilepsy can be attributed to AED noncompliance [58]. There is evidence suggesting that HIV is a significant risk factor for developing SE, with a multicentric crosssectional study exploring the association between convulsive SE among HIV+ individuals with active convulsive epilepsy in South Africa, Uganda, and Kenya reporting an adjusted odds ratio ([aOR] 2.1; 95\% CI 1.04-4.31) [59].

Also, SE can pose as a significant risk to both mother and fetus [60].

Of the six articles retrieved on SE and HIV, there was no study assessing the burden of SE in a HIV+ population on HAART. We retrieved only one article on AED adherence in HIV+ adults on HAART. One cohort study in Zambia [35] reported a suboptimal adherence at 6 months, resulting in 18 out of 33 (56\%) participants on HAART experiencing greater than 1-week lapse in AED supply, with participants reporting increased nausea or vomiting compared with baseline $(p<0.05)$.

\section{SJS/TEN}

There were no studies examining the impact of concomitant use of HAART and AED on SJS/TEN in pregnant HIV+ women in SSA.

SJS/TEN, AED, HAART, and pregnancy interact. It is well established that HIV+ persons have a higher incidence of SJS/TEN [61] with evidence suggesting poorer fetal outcomes in HIV+ women with SJS/TEN [36]. Treatment with commonly used AEDs such as carbamazepine, phenytoin, phenobarbital, and lamotrigine is considered to increase the risk of SJS or TEN [62]. A meta-analysis reported an incidence of skin rash with lamotrigine of almost $10 \%$ of persons from prospective studies [63] from high-income countries (HIC). Similarly, a recent review suggested that HAART, being implicated in $90 \%$ of cases, constitutes the most common putative medications in pregnant women [64].

The prevalence of the allele, human leukocyte antigen B gene $* 15: 02$, which is strongly associated with an increased risk of SJS and TEN, in patients taking carbamazepine $[65,66]$ and related drugs, including phenytoin and lamotrigine [67], is higher in people of African origin than in Caucasians (0.21\% versus $0.001 \%$, respectively) (www.allelefrequencies.net). Consequently, it is possible that the risk of SJS/TEN associated with these AEDs in SSA may be higher.

We retrieved 109 articles examining SJS/TEN in HIV+ individuals, of which five retrospective studies [37-41] and a prospective study were eligible [36]. No meta- 


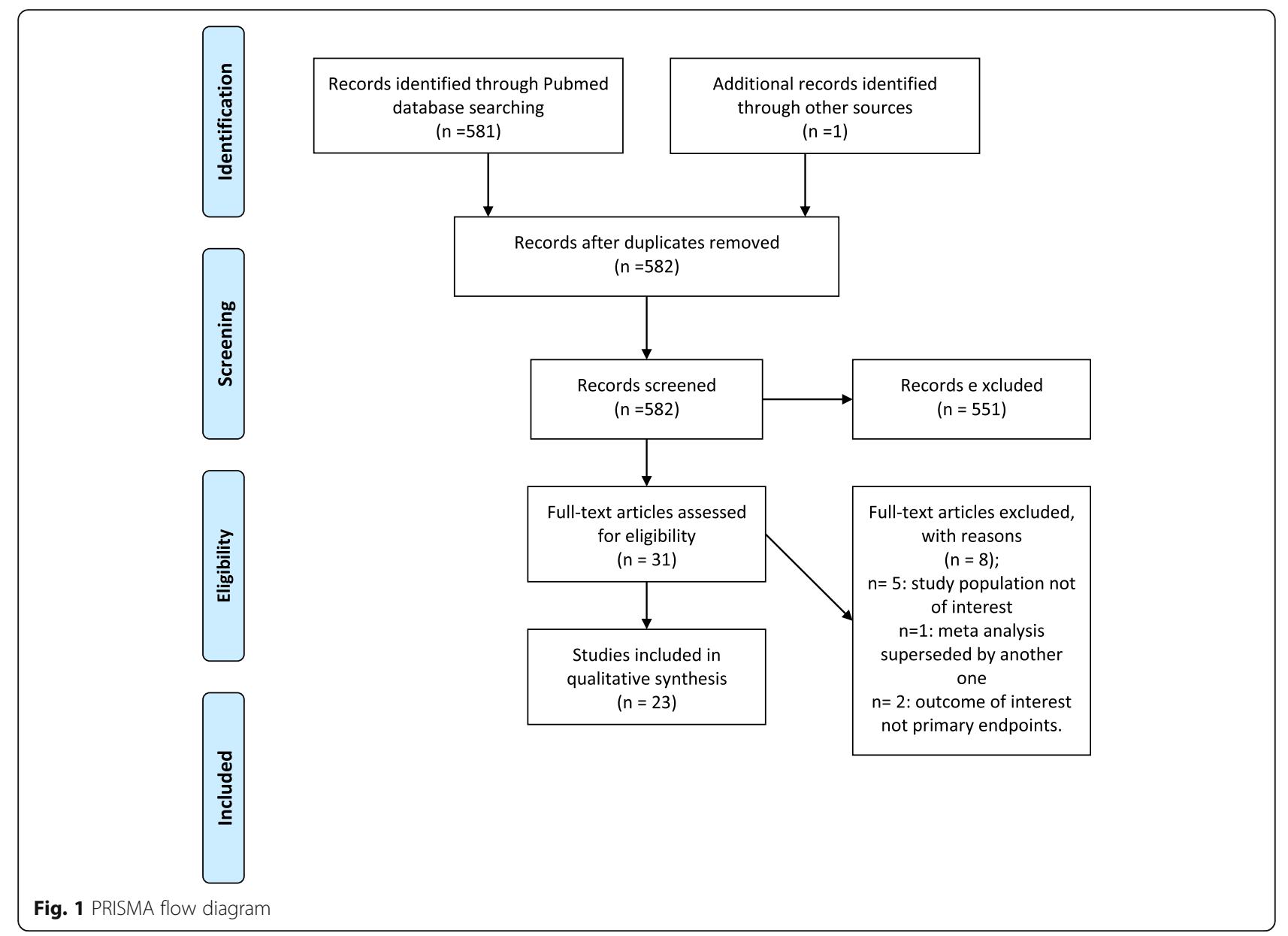

analysis was retrieved on the burden of SJS/TEN in HIV+ individuals on HAART or without in SSA. Three retrospective studies found nevirapine to be among the most common precipitants [37-39].

Two retrospective studies $[40,41]$ and a prospective study [36] examined data in HIV+ pregnant women on HAART. A matched case control study in South Africa reported that pregnancy increased the chances of developing SJS 14-fold (OR 14.28; 95\% CI 1.54-131.82) [40]. While a retrospective cohort study from a SSA reported that only $1.1 \%$ of the $703 \mathrm{HIV}+$ women on nevirapine developed SJS [41], in a prospective cohort study of pregnant HIV+ women in South Africa, nevirapine was found to be the offending drug in $95 \%$ of cases [36].

\section{Dyslipidemia}

No studies were retrieved assessing dyslipidemia among pregnant HIV+ WWE on HAART and AEDs in SSA.

Dyslipidemia, pregnancy, AED, and HAART intersect. While it was assumed that dyslipidemia in pregnancy is physiological and non-atherogenic [68], there is a mounting body of evidence suggesting an association with increased risks of preterm delivery $[69,70]$, gestational diabetes [71], and preeclampsia [72, 73]. Pregnant WWE on EI-AEDs may be at higher risk of dyslipidemia with studies from HIC suggesting an association with carbamazepine use [74-78]. Similarly, HAART has been implicated in dyslipidemia [79-81] during pregnancy, and especially and PI intake [82].

Seventy-five articles exploring dyslipidemia in HIV+ individuals on HAART were retrieved, of which one meta-analysis [42] and a retrospective study [43] were eligible. A meta-analysis estimated the overall prevalence of total cholesterol concentrations $(25.47 \% ; 95 \%$ CI 19.41-32.03\%) in the HIV+ population on HAART, low HDL cholesterol (45.6\%; 95\% CI 34.11-57.30\%), and elevated LDL cholesterol (24.30\%; 95\% CI 14.71-35.38\%) and triglycerides (19.68\%; 95\% CI 14.77-25.09) [42].

Only one study studied HAART use in pregnant women. A matched case control study of 154 pregnant and 151 non-pregnant on HAART in Nigeria reported a higher mean of very-low-density lipoprotein milligrams per deciliter in pregnant women 67.79 (SD 162.75) versus non-pregnant women $(p=0.045)$ [43]. 


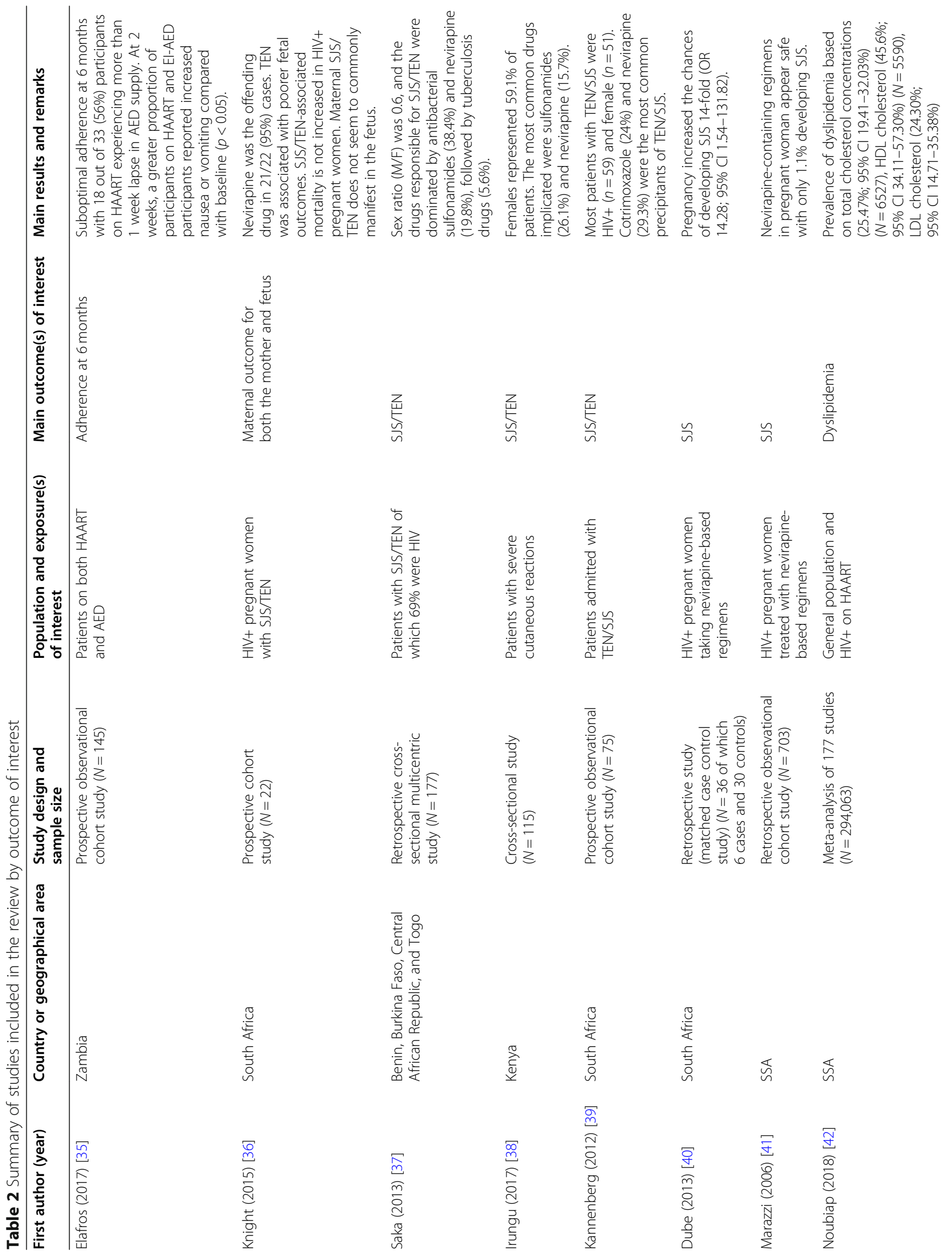




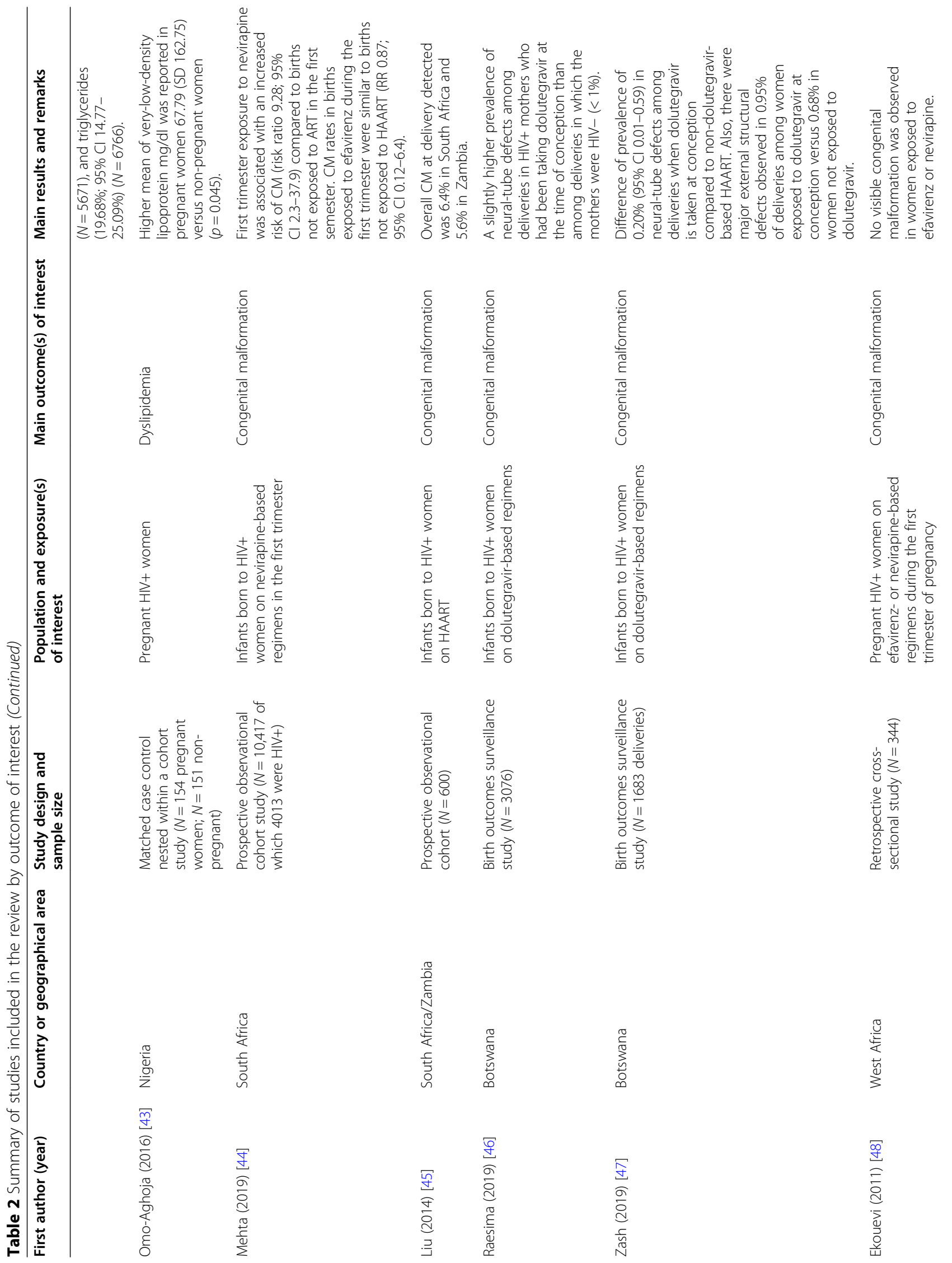




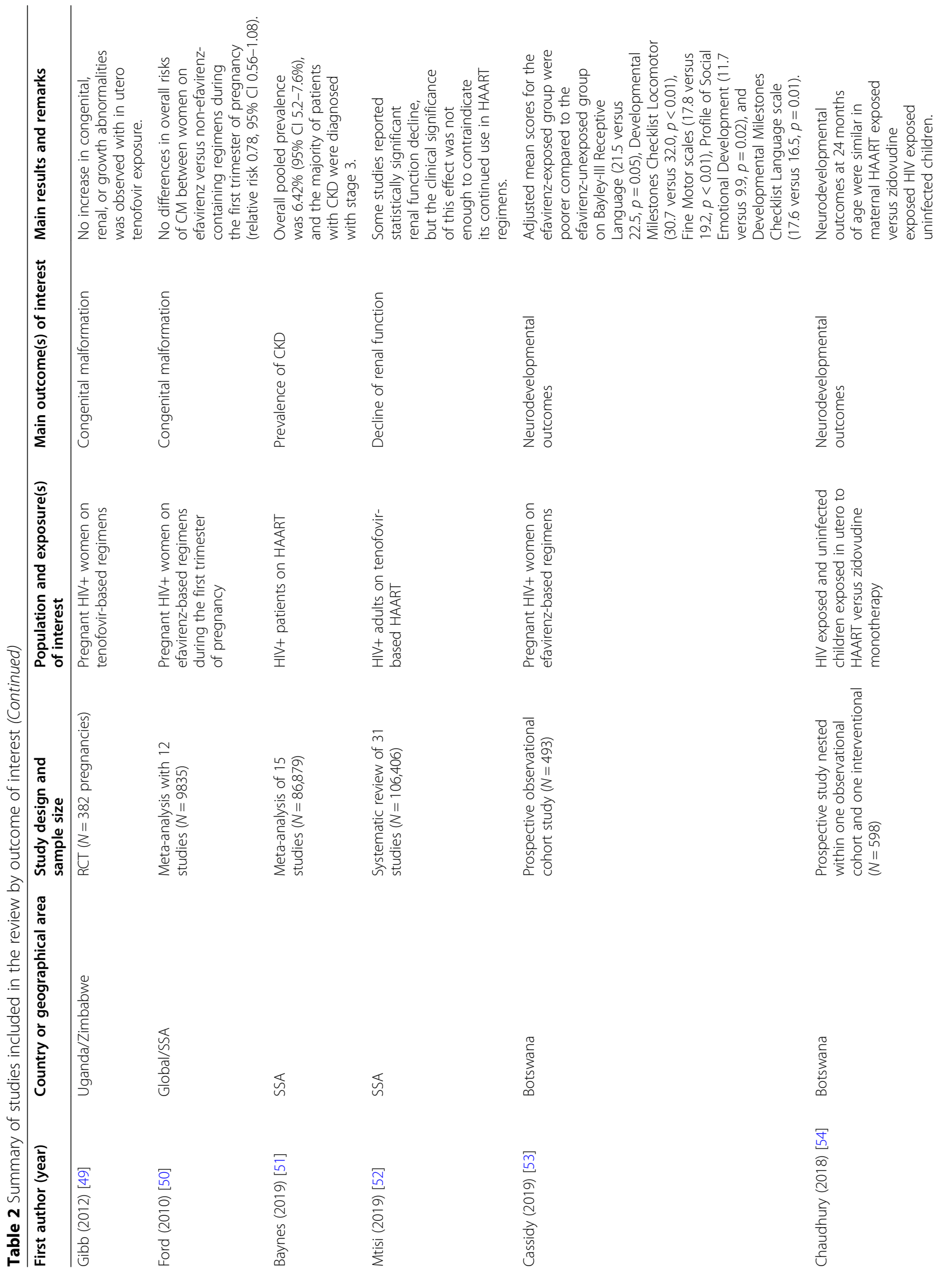




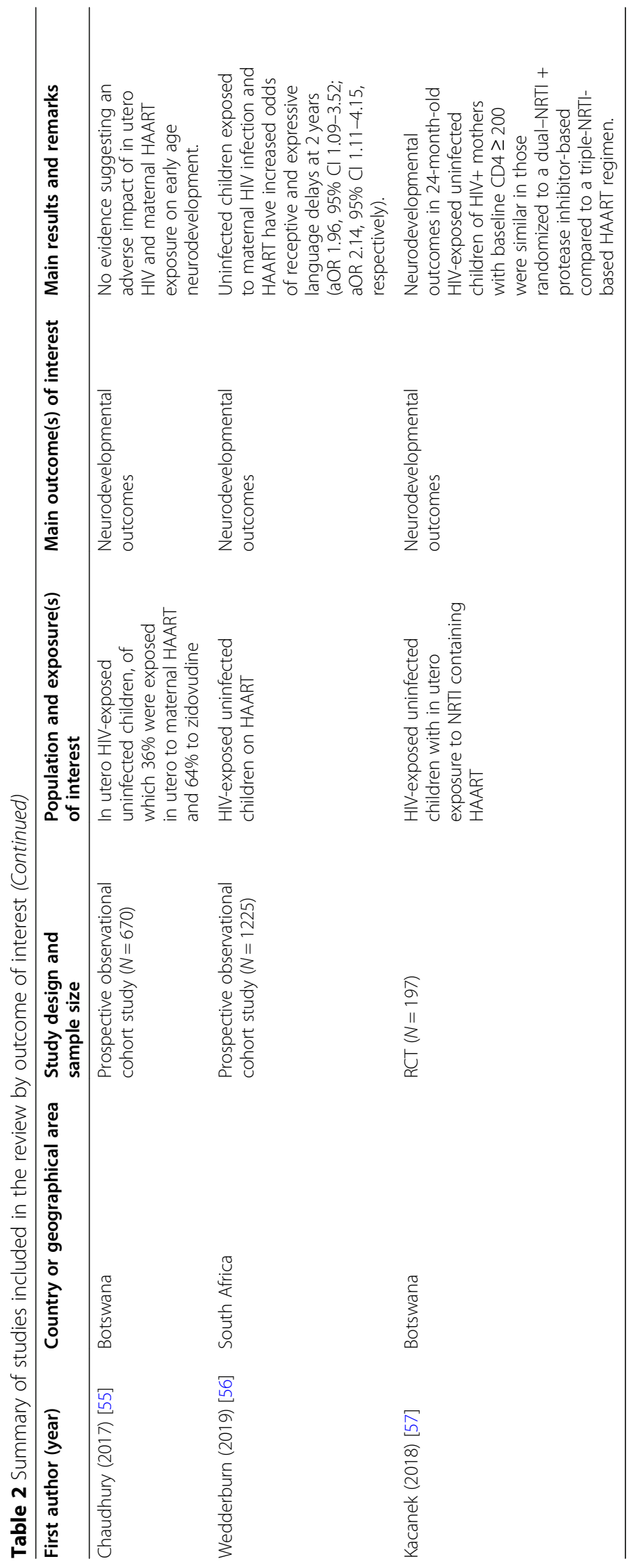




\section{Congenital malformation}

No study was identified reporting on CM among HIV+ WWE on HAART and AEDs in SSA.

CM, pregnancy, AED, and HAART intersect. Pregnant $\mathrm{HIV}+\mathrm{WWE}$ on AEDs may be at risk for CM. Different antiepileptic drugs and dosages have different teratogenic risks [30]. In contrast, a meta-analysis on the safety of perinatal HAART did not report an increased risk of CM [83], although further studies are warranted [84].

We retrieved 95 articles which examined CM related to HAART exposure in SSA, of which four prospective studies [44-47], one retrospective study [48], one RCT [49], and a meta-analysis [50] were eligible.

There was no meta-analysis on the burden of CM in $\mathrm{HIV}+$ pregnant women on HAART. The evidence for a higher risk of $\mathrm{CM}$ associated with nevirapine appears inconclusive. A study in South Africa found that first trimester exposure to nevirapine was associated with an increased risk of CM (risk ratio 9.28; 95\% CI 2.3-37.9) when compared to no exposure [44]. A HAART registry in South Africa reported a prevalence of major CM of $6.4 \%$ [45], within the ranges of the general population (95\% CI 2.5-8\%) [85].

In SSA, efavirenz forms the preferred first-line HAAR $\mathrm{T}$ [86]. A meta-analysis found no differences in overall risks of CM between women on efavirenz versus nonefavirenz-containing regimens during the first trimester of pregnancy (relative risk 0.78; 95\% CI 0.56-1.08) [50]. In line with these findings is a retrospective study, where no visible $\mathrm{CM}$ was observed in women exposed to either efavirenz or nevirapine [48]. In South Africa, a study showed that CM rates in births exposed to efavirenz during the first trimester were similar to births not exposed to HAART [44]. Moreover, a RCT among Ugandan/Zimbabwean HIV+ women did not observe any CM with in utero tenofovir exposure [49].

Nevertheless, amidst the transitioning to dolutegravirbased first-line regimens, there have been concerns about its safety in pregnancy. A preliminary analysis in Botswana suggested a higher prevalence of neural-tube defects with dolutegravir treatment at conception than with non-dolutegravir HAART at conception (difference $0.20 \%$; $95 \%$ CI $0.01-0.59 \%$ ) or with other types of HAAR $\mathrm{T}$ exposure [47]. A final analysis covering 90\% of all births in Botswana suggested dolutegravir exposure to be less than $1 \%$ [46].

\section{Chronic kidney disease}

No study examining the renal function of pregnant HIV+ WWE on HAART and AED in SSA was identified.

CKD, pregnancy, AED, and HAART intersect. Due to a suboptimal capacity of renal elimination, the dosing of AED in patients with CKD deserves special attention
[87], especially with levetiracetam, which is nearly $100 \%$ cleared by renal excretion [88]. Women with CKD are less able to make the renal adaptations needed for a healthy pregnancy [89], which is all the more problematic as a pregnancy is already associated with hormonal changes that directly and indirectly alter renal function [90]. CKD occurs both as a frequent complication of HIV infection and as a consequence of HAART and its complications [91], particularly in the patients on tenofovir [92], a commonly used in SSA [93], and which is a first-line regimen for all HIV+ pregnant and breastfeeding women initiating lifelong HAART independent of CD4+ count [94]. If not managed, HIV-associated kidney disease may progress to renal failure, which is a main predictive factor of complications in pregnant women [95].

Two hundred and five articles examined CKD in HIV+ individuals on HAART, of which one metaanalysis [51] and a systematic review [52] were eligible. The pooled prevalence of CKD among HIV+ individuals on HAART was estimated to be $6.42 \%$ (95\% CI $5.2-$ 7.6\%) [51].

There was no study examining risk factors for CKD in pregnant women on HAART in SSA. No studies were retrieved on CKD in pregnant women on HAART, and the only evidence stems from a systematic review reporting on tenofovir-based regimens in a general population, concluding that its clinical significance was not enough to contraindicate its continued use [52].

\section{Neurological development}

No study examining the risk of neurological disorders in children having been exposed in utero to a combination of HAART and AED in SSA was retrieved.

Neurological disorders, AEDs, HAART, and pregnancy intersect. As AEDs are able to cross the placenta, they may be associated with adverse neurodevelopment outcomes [31]. A meta-analysis of 29 observational cohorts carried out in HIC found valproate alone or in combination with another AED to be associated with the highest odds of adverse neurodevelopmental outcomes compared to women who did not receive AED during pregnancy (OR 7.40; 95\% CI 3.00-18.46) [31]. Furthermore, among the first and second generation broadspectrum AEDs targeting adults, lamotrigine and valproate (OR 8.88, 95\% CI 1.28-112.00; OR 17.29, 95\% CI $2.40-217.60$, respectively) were associated with an increased occurrence of autism compared to the same controls [31]. As endemic undernutrition and coinfections may compound any potential adverse effect of maternal HAART exposure, its potential effect in SSA is unclear [96].

We retrieved 50 articles on neurological disorders related to HAART in SSA, of which five prospective 
studies [53-57] were eligible. No meta-analyses were retrieved reporting on the burden on neurological disorders following in utero exposure to HAART. Different endpoints and conflicting data were found regarding the impact of in utero exposure to maternal HAART. One cohort study found HIV-exposed/uninfected children exposed in utero to efavirenz-based HAART to be at higher risk for neurodevelopmental disorders than HIVexposed/uninfected children exposed to non-efavirenzbased HAART [53]. Another cohort study suggested HIV-exposed/uninfected children exposed to HAART were at increased odds of receptive and expressive language delays at 2 years of age [56]. In contrast, a RCT study using different control groups (dual-NRTI + PIbased versus a triple-NRTI-based HAART regimen [57]) and two observational cohort studies (maternal HAAR T-exposed children versus HIV unexposed uninfected children [55] and HIV-exposed uninfected children exposed in utero to HAART versus zidovudine monotherapy [54]) did not exhibit significant differences in neurodevelopmental outcomes.

\section{Discussion}

Our review underscores the absence of studies related to our PICO population and the paucity of studies related to HIV+ pregnant women. The pooled burden of all six clinical aspects was not estimated for the HIV+ pregnant population (on HAART or without) and, for the general $\mathrm{HIV}+$ population on HAART, was absent for SE, SJS, $\mathrm{CM}$, and neurological outcomes. Furthermore, there were no studies retrieved reporting on CKD in pregnant HIV + HAART. This review highlights the need for a better understanding of clinical responses to first-line HAART in pregnant women in SSA and how the concomitant use of HAART and first-line/second-line AED may affect pregnancy, fetal, and neurological outcomes.

With high AED treatment gaps in SSA as well as suboptimal AED adherence, it is likely that HIV+ WWE exhibit significant fluctuations in therapeutic ranges, which may result in SE [58], hence rendering management of epilepsy in pregnant HIV+ women on HAART a clinical conundrum.

The maintenance of an optimal plasma concentration within the confinements of a therapeutic range is further complicated by the physiological changes experienced by WWE during pregnancy that may alter the pharmacokinetics of AEDs [97].

Levetiracetam, which has the best safety profile for $\mathrm{HIV}+$ women [10], is unaffordable for many, and valproate, which is contraindicated during pregnancy, is used as a last resort when other AEDs have failed to control seizures [98]. Switching from branded AEDs to generic AEDs, which may alter the absorption parameters of the drug along with its plasma level, is discouraged due to mounting evidence suggesting an association with poor outcomes [99].

It may be posited that switching between different first-line and second-line AEDs may entail larger fluctuations in pharmacokinetics. However, if a HIV+ WWE enters her pregnancy treated with valproic acid, it may still be clinically judicious to switch to a less teratogenic second-line AED as soon as possible provided that a gradual buildup of the added drug before weaning down the dose of the initial drug is ensured, while considering the unique physiological changes of pregnant WWE.

As EI-AEDs and lamotrigine use have been shown to entail a higher risk of SJS/TEN, to ensure safe management of seizures in HIV+ women, it is pivotal to assess the accrued risk due to HAART uptake. With the phasing out of nevirapine from HAART protocols, one can expect a reduction in the incidence of SJS/TEN in pregnant HIV+ WWE. Studies are required to determine if the severity of SJS/TEN impacts maternal and/or fetal outcomes and develop a validated SIS/TEN severity-ofillness score for this population, which should also consider the drugs implicated in SJS/TEN and commonly used by HIV+ individuals on HAART, including antibiotics [100], cotrimoxazole [39], malarial drugs [100], TB drugs [37, 39], and sulphonamides [37, 38, 100].

In utero exposure to valproic acid carries an unacceptable risk of CM and along with lamotrigine appears to carry a strong risk of autism, greatly augmented when used in combination [31]. More homogenous studies with similar endpoints are required to corroborate the emerging evidence of a higher risk of $\mathrm{CM}$ and possibly neurological impairment that dolutegravir and first-line HAART drugs may entail. It is also important to consider that in SSA, a non-negligible proportion of seizures may be partial or focal in nature; most of the latter being attributable to central nervous infections [101], which can cause structural damage in the brain. Such seizures have a varying response to AED treatment and can even be drug resistant, particularly in individuals with temporal abscesses [102]. Consequently, increasing the AED dose or switching to polytherapy in attempt to control refractory focal seizures may entail a heightened risk of teratogenicity as congenital malformations have been shown to increase in an AED dose-dependent and synergistic manner [103, 104].

The burden of dyslipidemia in the $\mathrm{HIV}+$ general population on HAART is high, and in pregnant women, HAART intake, especially PI-based regimens, along with EI-AED intake is likely to conspire to increase the risk for maternal dyslipidemia. In this regard, the benefits of statins, known to reduce progression of vascular disease, in HIV+ adults on HAART [105] should be weighed against findings showing no clear relationship of CM with its use but cautioning against use in pregnancy [106]. 
The burden of CKD in the HIV+ general population on HAART is non-negligible and likely to be higher in pregnant women, in whom renal function decline may be accelerated. In the absence of studies reporting on renal parameters in this population, findings on the safety of tenofovir can only be extrapolated from a general HIV population in SSA [52] and from studies on pregnant women from HIC suggesting that there were no significant effects of its exposure on renal function [107]. In SSA, proper management of seizures in pregnant HIV+ WWE requires knowledge of the epidemiology of HIV co-infections in the region and of potential interactions with AED. Treating pregnant HIV+ WWE entails the challenge of considering potential pharmacokinetic interactions between HAART, AEDs, anti-malarial and TB drugs, and cotrimoxazole when determining efficacious and safe dose adjustments, while also considering their changing kidney physiology.

A case for enhanced availability of 2nd-line AED can be made on public health grounds due to the potential of EIAED to lead to HAART-resistant strains [108], which may be transmitted vertically also through breastfeeding [109]. In addition, their potential interactions with other antiinfective treatments may lead to drug resistance within the community. Thus, the ultimate public health goal in SSA should be to treat all HIV+ women of reproductive age with epilepsy with levetiracetam.

In light of the scarcity of specific evidence-based epilepsy management guidelines, prevalence studies and cohort studies following HIV+ WWE over time and across pregnancies are needed to capture the cumulative effect of treatments in all identified clinical aspects, including barriers for concomitant HAART and AED adherence. This requires training of healthcare professionals in the management of SJD/TEN, renal function, dyslipidemia, and CM. Furthermore, operational research could bring insights into how enlarged PMTCT programs would be capable of accommodating epilepsy management.

\section{Strengths and limitations}

The wide breadth of this scoping review captures the evidence and knowledge gaps which had hitherto not yet been synthesized. However, we acknowledge a number of limitations. No papers were found concerning the pre-specified target population. In light of the preponderance of studies examining clinical outcomes in a general HIV population on HAART, along with a heterogeneity of study designs and controls, a cautionary approach should be employed when extrapolating findings to our study population to develop contextualized, evidence-based clinical guidelines.

\section{Conclusions}

There is a marked absence of studies for our PICO study population despite the urgency for evidenced-based clinical management guidelines for pregnant HIV+ WWE in SSA. Insights into how interactions between first- and second-line AEDs and newer antiretroviral agents play out in determining pregnancy and fetus outcomes should be sought, in particular how it impacts CKD, dyslipidemia, and CM. Efforts should be targeted at developing a validated SJS/TEN severity-of-illness score and a better understanding of the barriers to AED adherence. In light of the HIV burden in SSA, and the public health impact of interactions between EI-AEDs and first-line HAART, clinical guidelines should advocate for secondline AED.

\section{Supplementary information}

Supplementary information accompanies this paper at https://doi.org/10. 1186/s12916-020-01799-0.

Additional file 1. Annex 1

Additional file 2. Annex 2 .

\section{Abbreviations}

AIDS: Acquired immune deficiency syndrome; aOR: Adjusted odds ratio; AED: Antiepileptic drug; CKD: Chronic kidney disease; Cl: Confidence interval; CM: Congenital malformation; El-AED: Enzyme-inducing antiepileptic drug; HAART: Highly active antiretroviral therapy; HIC: High-income countries; HDL: High-density lipoprotein; HIV: Human immunodeficiency virus; LDL: Low-density lipoprotein; LIC: Low-income countries; NRTI: Nucleoside reverse transcriptase inhibitor; PI: Protease inhibitors; RCT: Randomized controlled trial; SJD: Stevens-Johnson syndrome; TEN: Toxic epidermal necrolysis; TB: Tuberculosis; WHO: World Health Organization; WWE: Women with epilepsy; SSA: Sub-Saharan Africa; PMTCT: Prevention of mother-to-child transmission

\section{Acknowledgements}

Natasha Zdraveska, clinical pharmacy consultant at specialized Hospital for Geriatric and Palliative Medicine, Skopje, Northern Republic of Macedonia.

\section{Authors' contributions}

SM the lead author conceived the manuscript, searched and screened the literature, synthesized the findings, interpreted the findings, and drafted the manuscript. LB interpreted the findings, and drafted and validated the final version of the manuscript. HM interpreted the findings and validated the final version of the manuscript. All authors read and approved the final manuscript.

\section{Funding}

This study did not receive any funding.

Availability of data and materials

Yes

Ethics approval and consent to participate $\mathrm{N} / \mathrm{A}$

Consent for publication

N/A

Competing interests

The authors declare that they have no competing interests. 


\section{Author details}

${ }^{1}$ Instiute of Tropical Medicine Antwerp, Antwerp, Belgium. ${ }^{2}$ Moi University/ Gynocare Fistula Centre, El Doret, Kenya.

Received: 26 May 2020 Accepted: 25 September 2020

Published online: 17 November 2020

\section{References}

1. World Health Organization, Geneva, Switzerland (2020); Key facts 2020. https://www.who.int/news-room/fact-sheets/detail/hiv-aids accessed online on October 2nd 2020.

2. UNAIDS, Geneva, Switzerland (2020); Global HIV \& AIDS statistics 2020 fact sheet. https://www.unaids.org/en/resources/fact-sheet accessed online on October 2nd 2020

3. UNAIDS, Geneva, Switzerland (2019) Fact sheet-World AIDS day https:// www.unaids.org/sites/default/files/media_asset/UNAIDS_FactSheet_en.pdf 2019 accessed online on October 2nd 2020.

4. AVERT, United Kingdom (2019) Programming/prevention/preventionmother-child transmission of HIV. https://www.avert.org/professionals/ hivprogramming/prevention/prevention-mother-child accessed online on October 2nd 2020

5. Deeks SG, Lewin SR, Havlir DV. The end of AIDS: HIV infection as a chronic disease. Lancet (London, England). 2013;382(9903):1525-33.

6. Sousa-Pinto B, et al. Stevens-Johnson syndrome/toxic epidermal necrolysis and erythema multiforme drug-related hospitalisations in a national administrative database. Clin Trans Allergy. 2018:8:2-2.

7. Ryom L, Mocroft A, Lundgren JD. Antiretroviral therapy, immune suppression and renal impairment in HIV-positive persons. Curr Opin HIV AIDS. 2014;9(1):41-7.

8. Islam FM, et al. Relative risk of cardiovascular disease among people living with HIV: a systematic review and meta-analysis. HIV Med. 2012; p. n/a-n/a.

9. Dillon DG, et al. Association of HIV and ART with cardiometabolic traits in sub-Saharan Africa: a systematic review and meta-analysis. Int J Epidemiol. 2013;42(6):1754-71.

10. Siddiqi O, Birbeck GL. Safe Treatment of Seizures in the Setting of HIV/AIDS. Curr Treat Options Neurol. 2013;15(4):529-43.

11. Ssentongo P. Prevalence and incidence of new-onset seizures and epilepsy in patients with human immunodeficiency virus (HIV): Systematic review and meta-analysis. Epilepsy Behav. 2019;93:49-55.

12. Fiest KM, et al. Prevalence and incidence of epilepsy: A systematic review and meta-analysis of international studies. Neurology. 2017:88(3):296-303.

13. Epilepsy, I.L.a., Definition of Epilepsy: A perspective for patients and caregivers 2014 ( https://www.llae.org/guidelines/definition-andclassification/the-2014-definition-of-epilepsy-a-perspective-for-patients-andcaregivers).

14. Garg RK. HIV infection and seizures. Postgrad Med J. 1999;75(885):387-90.

15. Chaudhry SA, et al. Human Immunodeficiency Viral Infection and Status Epilepticus in United States (2002-2009). J Vasc Interv Neurol. 2015;8(3):56-61.

16. World Health Organization (2019) Geneva, Switzerland; Epilepsy, key facts, 2019. https://www.who.int/news-room/fact-sheets/detail/epilepsy accessed online on October 2nd 2020

17. World Health Organization, Geneva, Switzerland (2019), Epilepsy 2019 https://www.who.int/news-room/fact-sheets/detail/epilepsy accessed online on October 2nd 2020

18. Meyer A-C, et al. Global disparities in the epilepsy treatment gap: a systematic review. Bull World Health Organ. 2010;88(4):260-6.

19. Ndoye NF, et al. Prevalence of epilepsy its treatment gap and knowledge, attitude and practice of its population in sub-urban Senegal an ILAE/IBE/ WHO study. Seizure. 2005;14(2):106-11.

20. Chin JH. Epilepsy treatment in sub-Saharan Africa: closing the gap. Afr Health Sci. 2012;12(2):186-92

21. Chisholm D. Cost-effectiveness of First-line Antiepileptic Drug Treatments in the Developing World: A Population-level Analysis. Epilepsia. 2005;46(5):751-9.

22. World Health Organization, Geneva, Switzerland (2018); Update on antiretroviral regimens for treating and preventing HIV infection and update on early infant diagnosis of HIV https://apps.who.int/iris/bitstream/handle/1 0665/273129/WHO-CDS-HIV-18.19-eng.pdf?sequence=1\&isAllowed=y accessed online on October 2nd, 2020.

23. Hikasa $\mathrm{S}$, et al. A potential drug interaction between phenobarbital and dolutegravir: A case report. J Infect Chemother. 2018;24(6):476-8.
24. Palazzo A, et al. Lower dolutegravir plasma concentrations in HIV-positive patients receiving valproic acid. J Antimicrob Chemother. 2017;73(3):826-7.

25. Birbeck GL, et al. Evidence-based guideline: Antiepileptic drug selection for people with HIV/AIDS: report of the Quality Standards Subcommittee of the American Academy of Neurology and the Ad Hoc Task Force of the Commission on Therapeutic Strategies of the International League Against Epilepsy. Neurology. 2012;78(2):139-45.

26. Hanaya R, Arita K. The New Antiepileptic Drugs: Their Neuropharmacology and Clinical Indications. Neurol Med Chir 2016:56(5):205-20.

27. World Health Organization, Geneva, Switzerland (2017) WHO model list of essential medicines. https://apps.who.int/iris/bitstream/handle/10665/273 826/EML-20-eng.pdf?ua=1 on October 2nd, 2020.

28. P.N, P., Antiepileptic Drug Interactions; Lamotrigine. p. pp 55-60.

29. Veroniki AA, et al. Comparative safety of anti-epileptic drugs during pregnancy: a systematic review and network meta-analysis of congenita malformations and prenatal outcomes. BMC Med. 2017;15(1):95.

30. Tomson T, et al. Comparative risk of major congenital malformations with eight different antiepileptic drugs: a prospective cohort study of the EURAP registry. Lancet Neurol. 2018;17(6):530-8.

31. Veroniki AA, et al. Comparative safety of antiepileptic drugs for neurological development in children exposed during pregnancy and breast feeding: a systematic review and network meta-analysis. BMJ Open. 2017;7(7):e017248.

32. Holdiness MR. Teratology of the antituberculosis drugs. Early Hum Dev. 1987;15(2):61-74.

33. Menon S, et al. Women with epilepsy in sub-Saharan Africa: A review of the reproductive health challenges and perspectives for management. Seizure. 2019:71:312-7.

34. Arksey H, O'Malley L. Scoping studies: towards a methodological framework Int J Soc Res Methodol. 2005;8(1):19-32.

35. Elafros MA, et al. Patient-Reported Adverse Effects Associated with Combination Antiretroviral Therapy and Coadministered Enzyme-Inducing Antiepileptic Drugs. Am J Trop Med Hyg. 2017;96(6):1505-11.

36. Knight $L$, et al. Stevens Johnson Syndrome and Toxic Epidermal Necrolysis: Maternal and Foetal Outcomes in Twenty-Two Consecutive Pregnant HIV Infected Women. PLoS One. 2015;10(8):e0135501.

37. Saka B, et al. Stevens-Johnson syndrome and toxic epidermal necrolysis in sub-Saharan Africa: a multicentric study in four countries. Int J Dermatol. 2013:52(5):575-9.

38. Irungu K, Nyamu D, Opanga S. Characterization of Stevens-Johnson Syndrome and Toxic Epidermal Necrolysis Among Patients Admitted to Kenyatta National Hospital: A Retrospective Cross-Sectional Study. Drugs Real World Outcomes. 2017;4(2):79-85.

39. Kannenberg SMH, et al. Toxic epidermal necrolysis and Stevens-Johnson syndrome in South Africa: a 3-year prospective study. QJM. 2012;105(9):839-46.

40. Dube N, Adewusi E, Summers R. Risk of nevirapine-associated StevensJohnson syndrome among HIV-infected pregnant women: The Medunsa National Pharmacovigilance Centre, 2007 - 2012. S Afr Med J. 2013;103(5): 322.

41. Marazzi MC, et al. Safety of nevirapine-containing antiretroviral triple therapy regimens to prevent vertical transmission in an African cohort of HIV-1infected pregnant women. HIV Med. 2006;7(5):338-44.

42. Noubiap JJ, et al. Prevalence of dyslipidaemia among adults in Africa: a systematic review and meta-analysis. Lancet Glob Health. 2018;6(9):e998e1007.

43. Omo-Aghoja LO, A.E, Omo-Aghoja WW, Onowhakpor A, Feyi-Waboso P. Is pregnancy associated with biochemical and haematological changes in HIVinfected Nigerian women? S Afr J Hiv Med. 2010;2010:45-8.

44. Mehta UC, et al. Birth outcomes following antiretroviral exposure during pregnancy: Initial results from a pregnancy exposure registry in South Africa. South Afr J HIV Med. 2019;20(1):971.

45. Liu KC, et al. Pregnancy outcomes and birth defects from an antiretroviral drug safety study of women in South Africa and Zambia. AIDS (London, England). 2014;28(15):2259-68.

46. Raesima MM, et al. Dolutegravir Use at Conception - Additional Surveillance Data from Botswana. N Engl J Med. 2019;381(9):885-7.

47. Zash R, et al. Neural-Tube Defects and Antiretroviral Treatment Regimens in Botswana. N Engl J Med. 2019;381(9):827-40.

48. Ekouevi DK, et al. Pregnancy outcomes in women exposed to efavirenz and nevirapine: an appraisal of the leDEA West Africa and ANRS Databases, Abidjan, Côte d'Ivoire. J Acquir Immune Defic Syndr (1999). 2011;56(2):183-7 
49. Gibb DM, et al. Pregnancy and infant outcomes among HIV-infected women taking long-term ART with and without tenofovir in the DART trial. PLoS Med. 2012;9(5):e1001217.

50. Ford N, et al. Safety of efavirenz in first-trimester of pregnancy: a systematic review and meta-analysis of outcomes from observational cohorts. Aids. 2010;24(10):1461-70.

51. Baynes $\mathrm{H}$, et al. Chronic kidney disease among human immunodeficiency virus positive patients on antiretroviral therapy in sub-Saharan Africa: A systematic review and meta-analysis. Saudi J Kidney Dis Transplant. 2019; 30(6):1190.

52. Mtisi TJ, et al. Tenofovir-associated kidney disease in Africans: a systematic review. AIDS Res Ther. 2019;16(1):12.

53. Cassidy AR, et al. In Utero Efavirenz Exposure and Neurodevelopmental Outcomes in HIV-exposed Uninfected Children in Botswana. Pediatr Infect Dis J. 2019:38(8):828-34.

54. Chaudhury $\mathrm{S}$, et al. In-utero exposure to antiretrovirals and neurodevelopment among HIV-exposed-uninfected children in Botswana. AIDS (London, England). 2018;32(9):1173-83.

55. Chaudhury S, et al. Neurodevelopment of HIV-Exposed and HIV-Unexposed Uninfected Children at 24 Months. Pediatrics. 2017;140(4):e20170988.

56. Wedderburn CJ, et al. Neurodevelopment of HIV-exposed uninfected children in South Africa: outcomes from an observational birth cohort study. The Lancet. Child Adolesc Health. 2019;3(11):803-13.

57. Kacanek D, et al. Pediatric Neurodevelopmental Functioning After In Utero Exposure to Triple-NRTI vs. Dual-NRTI + PI ART in a Randomized Trial, Botswana. J Acquir Immune Defic Syndr (1999). 2018;79(3):e93-e100.

58. Neligan A, Shorvon SD. Frequency and Prognosis of Convulsive Status Epilepticus of Different Causes. Arch Neurol. 2010;67(8).

59. Kariuki SM, et al. Prevalence and factors associated with convulsive status epilepticus in Africans with epilepsy. Neurology. 2015;84(18):1838-45.

60. Rajiv KR, Radhakrishnan A. Status epilepticus in pregnancy: Etiology, management, and clinical outcomes. Epilepsy Behav. 2017;76:114-9.

61. Knight $\mathrm{L}$, et al. Factors associated with increased mortality in a predominantly HIV-infected population with Stevens Johnson syndrome and toxic epidermal necrolysis. PLoS One. 2014;9(4):e93543.

62. Gidal BE. Carbamazepine hypersensitivity: progress toward predicting the unpredictable. Epilepsy Curr. 2011;11(6):189-91.

63. Wang $X-q$, et al. Risk of a lamotrigine-related skin rash: Current metaanalysis and postmarketing cohort analysis. Seizure. 2015;25:52-61.

64. Sharma AN, et al. Stevens-Johnson syndrome and toxic epidermal necrolysis in pregnant patients: A systematic review. Int J Womens Derm. 2020.

65. Chung W-H, et al. A marker for Stevens-Johnson syndrome. Nature. 2004; 428(6982):486

66. Yip VL, et al. HLA Genotype and Carbamazepine-Induced Cutaneous Adverse Drug Reactions: A Systematic Review. Clin Pharm Ther. 2012;92(6): 757-65.

67. Fan WL, et al. HLA Association with Drug-Induced Adverse Reactions. J Immunol Res. 2017;2017:3186328.

68. Bartels $\ddot{A}$, O'Donoghue K. Cholesterol in pregnancy: a review of knowns and unknowns. Obstet Med. 2011:4(4):147-51.

69. Edison RJ, et al. Adverse birth outcome among mothers with low serum cholesterol. Pediatrics. 2007;120(4):723-33.

70. Maymunah $\mathrm{AO}$, et al. Hypercholesterolaemia in pregnancy as a predictor of adverse pregnancy outcome. Afr Health Sci. 2014;14(4):967-73.

71. González-Clemente JM, et al. Increased cholesterol intake in women with gestational diabetes mellitus. Diabetes Metab. 2007;33(1):25-9.

72. Qiu C, et al. Oxidized low-density lipoprotein (Oxidized LDL) and the risk of preeclampsia. Physiol Res. 2006;55(5):491-500.

73. Sanchez SE, et al. A case-control study of oxidized low density lipoproteins and preeclampsia risk. Gynecol Endocrinol. 2005;21(4):193-9.

74. Eirís J, et al. The effects on lipid and apolipoprotein serum levels of longterm carbamazepine, valproic acid and phenobarbital therapy in children with epilepsy. Epilepsy Res. 2000;41(1):1-7.

75. Castro-Gago M, et al. Evolution of Serum Lipids and Lipoprotein (a) Levels in Epileptic Children Treated With Carbamazepine, Valproic Acid, and Phenobarbital. J Child Neurol. 2006;21(1):48-53.

76. Verrotti $A$, et al. Changes in serum lipids and lipoproteins in epileptic children treated with anticonvulsants. J Paediatr Child Health. 1997;33(3): 242-5.

77. Verrotti A, et al. Serum lipids and lipoproteins in patients treated with antiepileptic drugs. Pediatr Neurol. 1998;19(5):364-7.
78. Voudris KA, et al. Early and persistent increase in serum lipoprotein (a) concentrations in epileptic children treated with carbamazepine and sodium valproate monotherapy. Epilepsy Res. 2006;70(2-3):211-7.

79. Feeney ER, Mallon PWG. HIV and HAART-Associated Dyslipidemia. Open Cardiovasc Med J. 2011;5:49-63.

80. Dillon DG, et al. Association of HIV and ART with cardiometabolic traits in sub-Saharan Africa: a systematic review and meta-analysis. Int J Epidemiol. 2016;45(6):2210-1.

81. Dimala CA, Blencowe $\mathrm{H}$, Choukem SP. The association between antiretroviral therapy and selected cardiovascular disease risk factors in subSaharan Africa: A systematic review and meta-analysis. PLoS One. 2018;13(7): e0201404.

82. Harmsen MJ, et al. The association between HIV (treatment), pregnancy serum lipid concentrations and pregnancy outcomes: a systematic review. BMC Infect Dis. 2017:17(1):489.

83. Veroniki AA, et al. Comparative safety and effectiveness of perinatal antiretroviral therapies for HIV-infected women and their children: Systematic review and network meta-analysis including different study designs. PLoS One. 2018;13(6):e0198447.

84. Williams PL, et al. Congenital anomalies and in utero antiretroviral exposure in human immunodeficiency virus-exposed uninfected infants. JAMA Pediatr. 2015;169(1):48-55.

85. Human Genetics Policy Guidelines for the Management and Prevention of Genetic Disorders, B.D.a.D.P., South Africa: Sub-Directorate, Human Genetics, National Department of Health 2008: p. www.doh.gov.za Accessed on line on 21 Mar 2020.

86. AIDSmap, Efavirenz does not increase the risk of suicidal thoughts or depression in Ugandan adults. 2018.

87. Bansal AD, Hill CE, Berns JS. Use of Antiepileptic Drugs in Patients with Chronic Kidney Disease and End Stage Renal Disease. Semin Dial. 2015; 28(4):404-12.

88. G.A, M. Clinical Challenges in Therapeutic Drug Monitoring; Special Populations, Physiological Conditions and Pharmacogenomics: Elsevier Sciencedirect; 2016. p. 101-34.

89. Williams D, Davison J. Chronic kidney disease in pregnancy. BMJ. 2008; 336(7637):211-5.

90. Hussein W, Lafayette RA. Renal function in normal and disordered pregnancy. Curr Opin Nephrol Hypertens. 2014;23(1):46-53.

91. Naicker S, Rahmanian S, Kopp JB. HIV and chronic kidney disease. Clin Nephrol. 2015;83(7 Suppl 1):32-8.

92. Mocroft A, et al. Cumulative and current exposure to potentially nephrotoxic antiretrovirals and development of chronic kidney disease in HIV-positive individuals with a normal baseline estimated glomerular filtration rate: a prospective international cohort study. Lancet HIV. 2016;3(1): e23-32.

93. Pintye J, et al. Maternal Tenofovir Disoproxil Fumarate Use During Pregnancy Is Not Associated With Adverse Perinatal Outcomes Among HIVinfected East African Women: A Prospective Study. J Infect Dis. 2017;216(12): $1561-8$

94. World Health Organization Geneva, Switzerland (2013); Consolidated guidelines on the use of antiretroviral drugs for treating and preventing HIV infection. https://www.who.int/hiv/pub/arv/arv-2016/en/ accessed online 2 Oct 2020.

95. Manisco G, et al. Pregnancy in end-stage renal disease patients on dialysis: how to achieve a successful delivery. Clin Kidney J. 2015;8(3):293-9.

96. Ramokolo V, et al. In Utero ART Exposure and Birth and Early Growth Outcomes Among HIV-Exposed Uninfected Infants Attending Immunization Services: Results From National PMTCT Surveillance, South Africa. Open Forum Infect Dis. 2017;4(4):ofx187.

97. Reisinger $\mathrm{TL}$, et al. Antiepileptic drug clearance and seizure frequency during pregnancy in women with epilepsy. Epilepsy Behav. 2013;29(1):13-8.

98. Voinescu PE, Pennell PB. Management of epilepsy during pregnancy. Expert Rev Neurother. 2015;15(10):1171-87.

99. Antiepileptic drugs: the drawbacks of generic substitution. Lancet Neurol. 2010;9(3):227

100. Fadeyibi 10 , et al. Severe idiosyncratic drug reactions with epidermal necrolysis: A 5-year study. Indian J Plast Surg. 2011;44(3):467-73.

101. Bittencourt PR, S.J, Mazer S Meinardi H, editor, Viral, bacterial, fungal and parasitic infections associated with seizure disorders. Elsevier Sciences; , 1999. Handbook of Clinical Neurology. Vol. 72. Amsterdam: 1999. The Epilepsies: p. pp. 145-174. 
102. OH DB. In: Engel JJ, Pedley TA, editors. Infections and inflammatory diseases: Lippincott Williams \& Wilkins; 2008. p. 2643-52. 2(Epilepsy).

103. Pennell PB. Too Complicated or So Simple: AED Type and AED Dose Matter for Pregnancy. Epilepsy Curr. 2012;12(2):63-5.

104. Morrow J, et al. Malformation risks of antiepileptic drugs in pregnancy: a prospective study from the UK Epilepsy and Pregnancy Register. J Neurol Neurosurg Psychiatry. 2006;77(2):193-8.

105. Eckard AR, McComsey GA. The role of statins in the setting of HIV infection. Curr HIV/AIDS Rep. 2015;12(3):305-12.

106. Karalis DG, et al. The risks of statin use in pregnancy: A systematic review. J Clin Lipidol. 2016;10(5):1081-90.

107. Mofenson LM, Baggaley RC, Mameletzis I. Tenofovir disoproxil fumarate safety for women and their infants during pregnancy and breastfeeding AIDS. 2017;31(2):213-32.

108. Fillekes $\mathrm{Q}$, et al. Effect of 7 days of phenytoin on the pharmacokinetics of and the development of resistance to single-dose nevirapine for perinatal HIV prevention: a randomized pilot trial. J Antimicrob Chemother. 2013; 68(11):2609-15.

109. Zeh C, et al. HIV-1 drug resistance emergence among breastfeeding infants born to HIV-infected mothers during a single-arm trial of triple-antiretroviral prophylaxis for prevention of mother-to-child transmission: a secondary analysis. PLoS Med. 2011;8(3):e1000430.

\section{Publisher's Note}

Springer Nature remains neutral with regard to jurisdictional claims in published maps and institutional affiliations.

Ready to submit your research? Choose BMC and benefit from:

- fast, convenient online submission

- thorough peer review by experienced researchers in your field

- rapid publication on acceptance

- support for research data, including large and complex data types

- gold Open Access which fosters wider collaboration and increased citations

- maximum visibility for your research: over $100 \mathrm{M}$ website views per year

At $\mathrm{BMC}$, research is always in progress.

Learn more biomedcentral.com/submissions 